\title{
Methods to achieve fast, accurate, and mechanically robust optical breadboard alignment
}

\author{
Martin Grenier,* Nichola Desnoyers, Frederic Lamontagne, \\ Bruno Leduc $\odot$, Mathieu Legros, and Simon Paradis \\ Institut National d'Optique, Quebec, QC, Canada
}

\begin{abstract}
Typical laboratory optical systems use commercially off-the-shelf components in which emphasis is oriented toward ease of assembly and a wide range of adjustability. However, these mounts often require individual alignments that, when each degree of adjustability is cumulated in a complex optical system, can be inefficient and time consuming. Furthermore, most of these optomechanical mounts lack the mechanical robustness required to maintain operational performances out of the laboratory environment. An optomechanical assembly method based on passively aligning design features is proposed to simplify breadboard level optical systems, to improve alignment accuracy and maintaining operational pointing stability. Given the recent improvements in lens passive centering techniques, it seemed worth exploring methods to reduce alignment time and improve the mechanical robustness of laboratory setups. Recent studies show that a typical optical lens centering of $<1$ arc min with respect to its mount can be achieved using patented auto centering and edge contact mounting technologies. To achieve similar position accuracy between multiple lenses on a portable breadboard, lens mounts should be designed and built with proper reference surfaces and a system should easily reference one mount with respect to the other. The use of reference spheres and dedicated optomechanical mounts is employed to leverage the standard threaded holes of laboratory breadboards and achieve precise lens mount positioning. A series of optomechanical mounts incorporating these techniques are therefore tested. Position accuracy and repeatability are measured mechanically with a coordinate measuring machine and optically with the active monitoring of a laser beam centroid position. Measured position accuracy at the optomechanical mount level is $<50 \mu \mathrm{m}$ with a repeatability of less than $5 \mu \mathrm{m}$ per interface. The optomechanical mounts robustness is tested within typical storage temperature range of $-46^{\circ} \mathrm{C}$ to $63^{\circ} \mathrm{C}$ and at vibrations levels exceeding typical shipping conditions. Measured optical pointing stability of a simple optical system after environmental testing was found to be under $25 \mu \mathrm{m}$. This method should be a promising solution to bridge the design technological gap between the early prototyping and the production phases. (C) The Authors. Published by SPIE under a Creative Commons Attribution 4.0 Unported License. Distribution or reproduction of this work in whole or in part requires full attribution of the original publication, including its DOI. [DOI: 10.1117/1.OE.60.5.051213]
\end{abstract}

Keywords: lens mounting; centering; alignment; edge mounting; QuickCTR-edge; QuickCTR; lens barrel; optomechanics; auto-centering; self-centering; optomechanical mount; optical mount; optomechanical stability.

Paper 20201439SS received Dec. 8, 2020; accepted for publication Mar. 9, 2021; published online Mar. 24, 2021.

\section{Introduction}

In the industry, the need in terms of optical component positioning accuracy may vary a lot from one application to the other. Meanwhile, according to Institut National d'Optique (INO)'s experience, most industrial optical systems fit within $\pm 50 \mu \mathrm{m}$ of lens centration. Nonetheless, almost all new optical systems require demonstration work done in the laboratory environment. The alignment of each lens or optical element of an optical system is often tedious and sometimes a difficult task that may last many hours up to multiple days of work. Moreover, it requires a lot of engineering work to achieve the state where the same optical design can be built to meet the

*Address all correspondence to Martin Grenier, martin.grenier@ino.ca 
mechanical stability required to withstand shipping conditions without losing its initial alignment settings, thereafter called as mechanical robustness.

While presently available breadboards and optomechanical mounts on the market are well designed to achieve maximum flexibility and ease of use, they all lack the same features: built-in precise positioning, position repeatability, and mechanical robustness. Typical commercially available lens and mirror mounts, such as optical posts, lens tubes, and cage systems, are made to be positioned with unprecise laboratory fixtures as they do not have external mechanical references. Therefore, breadboard and table top optomechanical mounts usually rely on visual alignment rather than mechanical referencing. Initial position accuracy of a lens mount that is visually aligned is not well described in literature. However, it could be reasonably assumed that it is possible to center at resolution of $1 / 20$ 'th of the clear aperture of the optomechanical mount without any special tooling. This corresponds to about $\pm 1 \mathrm{~mm}$ in translation $(X, Y, Z)$ for a $25.4 \mathrm{~mm}$ diameter lens mount. Using a similar approximation for the angular position of the mount, $\mathrm{a} \pm 2 \mathrm{deg}$ in rotation along the post axis (yaw) can be estimated due to the visual nature of the positioning made in the laboratory. Depending on the experiment sensitivity to misalignment, this initial optomechanical mount positioning is then refined through an iterative optimization process that requires the use of different techniques, tools such as targets, pin holes, wavefront sensors, point source microscopes, and the use of multiple linear translation and rotation stages to overcome the error buildup. By experience, after a first optimization process, it is not uncommon to find single optical elements decentered by $0.5 \mathrm{~mm}$ and tilted by $0.5 \mathrm{deg}$. Identifying misplaced optical elements and fine tuning their position to correct these last hundreds of microns can be time consuming. In addition, the lens position in its cell relies more on axial play between the cell thread and the lens outer diameter. Consequently, even after lens position optimization, if a lens must be removed for cleaning, inspection, or any other reason, there is a high probability that the lens will not return to its original position after reassembly. It implies that rework will be required on the optical system when a lens is removed.

It is also worth noting that most commercially available translation and angular adjustable stages are not designed to survive mechanical shocks and vibration that would be encountered in commercial shipping conditions. These adjustable stages are usually heavy, fragile, and expensive; therefore, it is not practical to use them in optical assemblies destined to be shipped. With the ever-increasing speed of product development to achieve fast time-to-market goals, there is a need for a prototyping method that can reduce alignment time using accurate positioning, high position repeatability while being mechanically robust enough to be used as a product and to survive shipping constraints.

A line of optomechanical mounts and breadboards was developed specifically for that purpose. It is designed to ease optical alignment by passively centering lenses and mirrors from 12.7 to $50.8 \mathrm{~mm}$ in diameter. High position and angular accuracies are expected without sacrificing robustness.

\section{Operation Principle}

A trivial method used for decades to achieve repeatable mount referencing is the use of dedicated locating pins on custom-designed support structures or benches. It is generally accepted that abutting a lens mount on locating pin/bushing assembly can typically achieve 10 to $25 \mu \mathrm{m}$ in position repeatability. ${ }^{1}$ However, adding reference pins on breadboards is not practical, as it requires implementing a second matrix of holes specifically for the pins. Since typical breadboards already include an evenly spaced grid of mounting threaded holes, it is tempting to leverage existing holes into proper referencing features. Usually, conventional threaded holes are not considered reliable interfaces for precision positioning. However, it is possible to overcome this issue by adding a slightly oversized entrance cone (or chamfer) at the border of the threaded holes on the breadboard or optical table. It is then possible to use this conical interface as a locating surface for precision reference balls. A sphere inserted in a cone locks the reference ball's three degrees of freedom (DOF) in translation, defining a single point in space for each mounting hole. The combination of two or three reference points can then be used to accurately locate lens or mirror mounts anywhere on the breadboard. The availability of known reference 


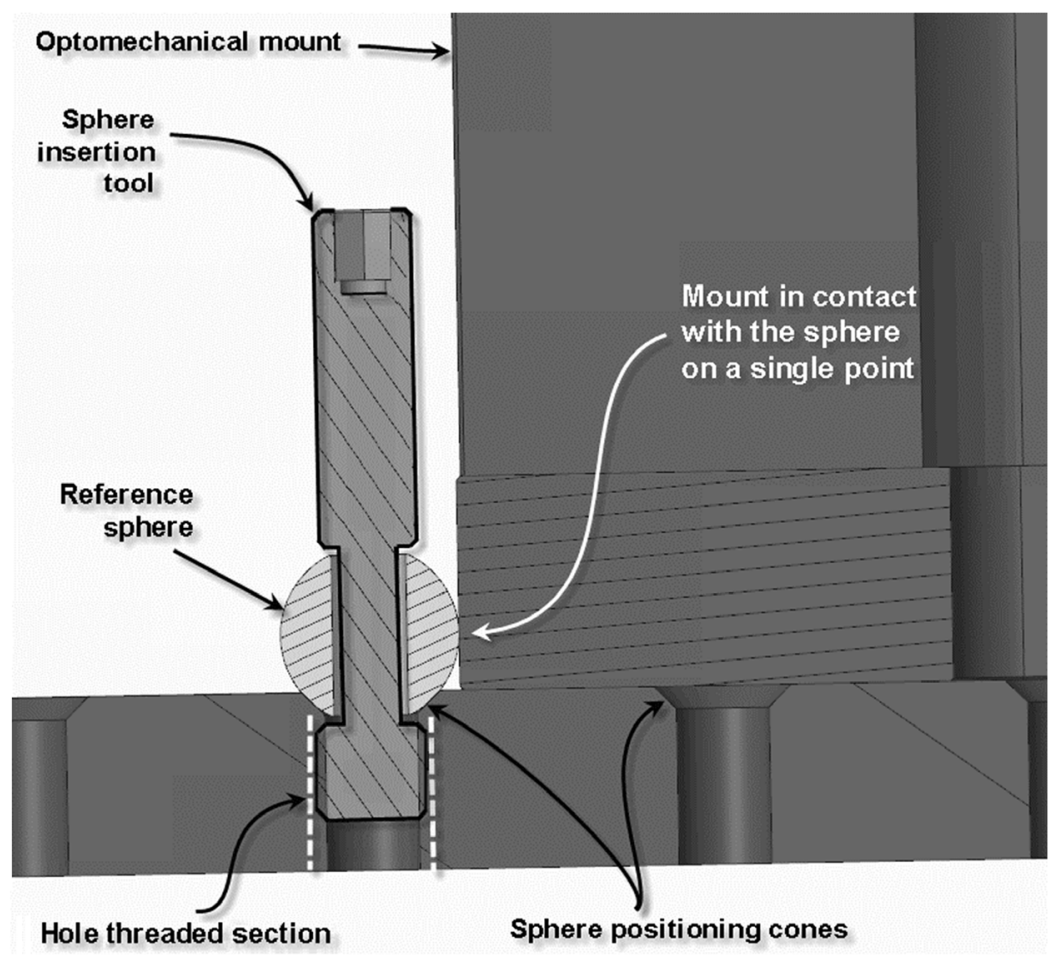

Fig. 1 Sphere reference tool in positioning cone principle.

points at close intervals opens the possibility to assemble an optical system mostly like building blocks: Inserting the optical element, placing the reference spheres where needed, abutting the lens mount on the spheres, locking the mount in position, removing the spheres, and repeating for the following one. Figures 1 and 2 show the ball referencing arrangement in a hole and the lens mount typical ball abutment arrangement, respectively.

The optomechanical mount six-DOF are defined in the coordinate system of Fig. 3. The breadboard mounting plane allows to fix three-DOF: vertical translation $(Z)$, pitch, and roll rotations. Then the three other DOF are to be defined with the spheres. The first one is the yaw rotation or rotation about a vertical $(Z)$ axis. The second and third are the breadboard planar

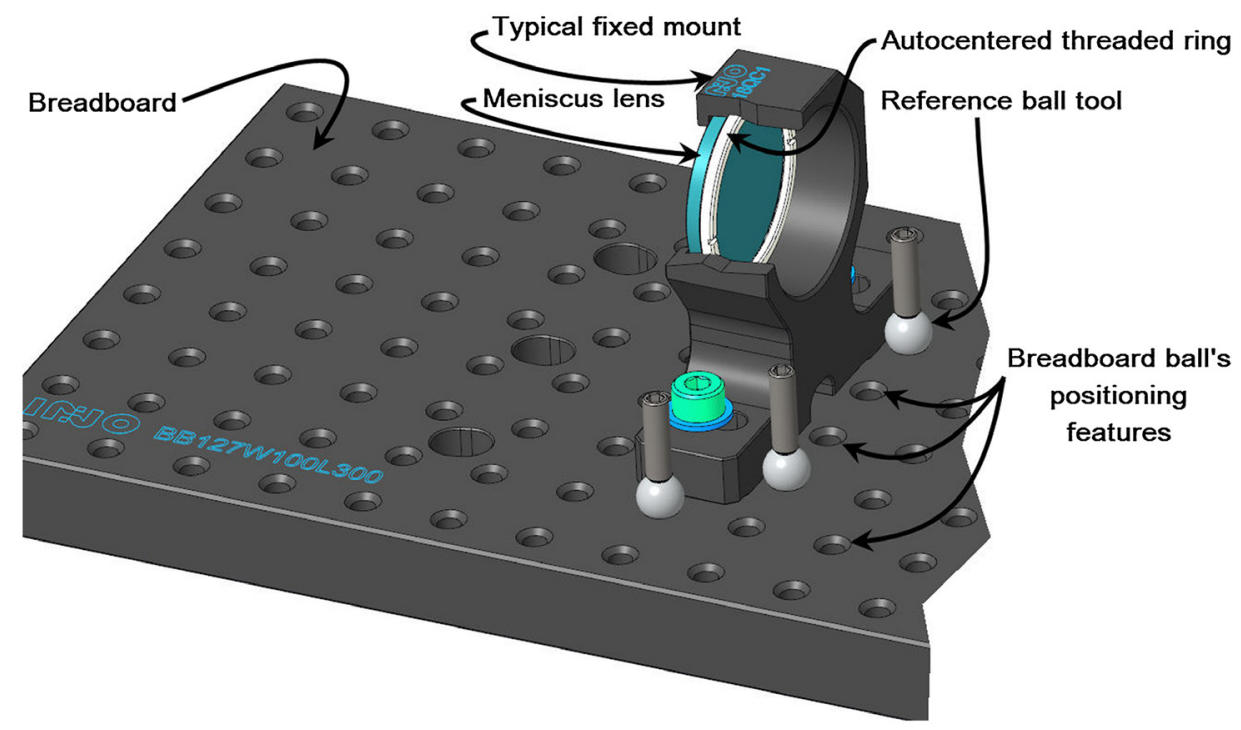

Fig. 2 Three-point contact positioning principle. 


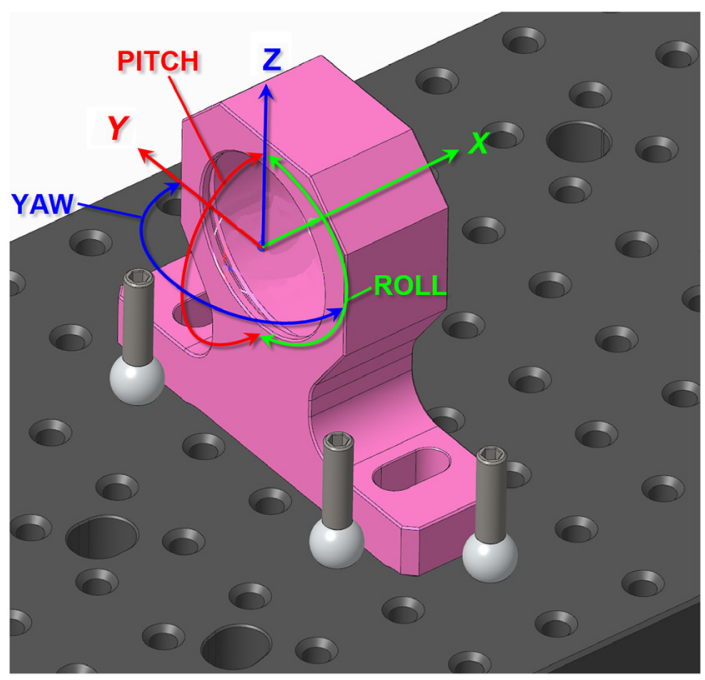

Fig. 3 Optomechanical mount coordinate system definition.

translations where axial refers to the direction parallel to the optical axis $(X)$ and lateral refer to the direction perpendicular to the optical axis $(Y)$.

The developed line of breadboard is based on M4 screws and 12.5-mm hole spacing to allow maximum flexibility in the lens optical layout. As shown in Fig. 1, the reference ball tool construction is essentially the assembly of a small M4 thumb screw containing a captive high precision ball. The M4 thumb screw insertion tool serves the dual purpose of locking the reference ball in position against the breadboard's reference cones and allows simpler ball manipulation in a lab environment. The $6.35-\mathrm{mm}$ diameter ball through hole fit is loose on the insertion tool to allow the ball to freely locate itself in the breadboard reference cone. At the insertion, the ball must be free to move onto the shaft to obtain the bests results. Otherwise, the ball position would be driven by the threaded hole, which is not as precise as the reference cone. Even a small, threaded hole perpendicularity error can prevent the ball to sit correctly in the precise reference cone thus reducing the ball position accuracy and repeatability.

Some of the advantages of using balls instead of pins to position lens mounts are reduced sensitivity to hole and lower chamfer perpendicularity errors, in addition to the ease of removal after the mount is locked in place. Angle errors for holes equally affects the pin and the reference sphere center's position assuming contact height of the sphere and pin height are the same. The sphere's contact height with respect to the breadboards surface can be described as

$$
h_{\text {SCH }}=\frac{\varnothing_{\text {Sphere }}}{2} \cdot \sin \left(\theta_{\text {Cone }}\right)-\left(\frac{\varnothing_{\text {Cone }}-\varnothing_{\text {Sphere }} \cdot \cos \left(\theta_{\text {Cone }}\right)}{2}\right) \cdot \cot \left(\theta_{\text {Cone }}\right),
$$

where $\varnothing_{\text {Sphere }}$ is the reference sphere diameter, $\theta_{\text {Cone }}$ is the entry cone half angle, and $\varnothing_{\text {Cone }}$ is the entry cone diameter on the breadboard top. The lateral shift of the sphere's center, or position error, due to a tilted entry hole is

$$
\sigma_{C T}=h_{S C H} \cdot \tan \left(\theta_{T i l t}\right)
$$

where $\sigma_{C T}$ is the sphere center position error due to the pin tilt angle, $\varnothing_{\text {Sphere }}$ is the reference sphere diameter, $\theta_{\text {Cone }}$ is the entry cone half angle, $\varnothing_{\text {Cone }}$ is the entry cone diameter on the breadboard top, and $\theta_{\text {Tilt }}$ is the cone or threaded hole angular tilt error. However, for the sphere, the resulting error will be limited to its center translation $\sigma_{C T}$ and the contact point on the mount will be mostly unchanged in all possible contact orientations. In comparison, abutting an optomechanical mount on a tilted pin from different directions provides contact points at different heights on an optomechanical mount, thus increasing the total position uncertainty. This abutting position difference due to a tilted pin can be described as 


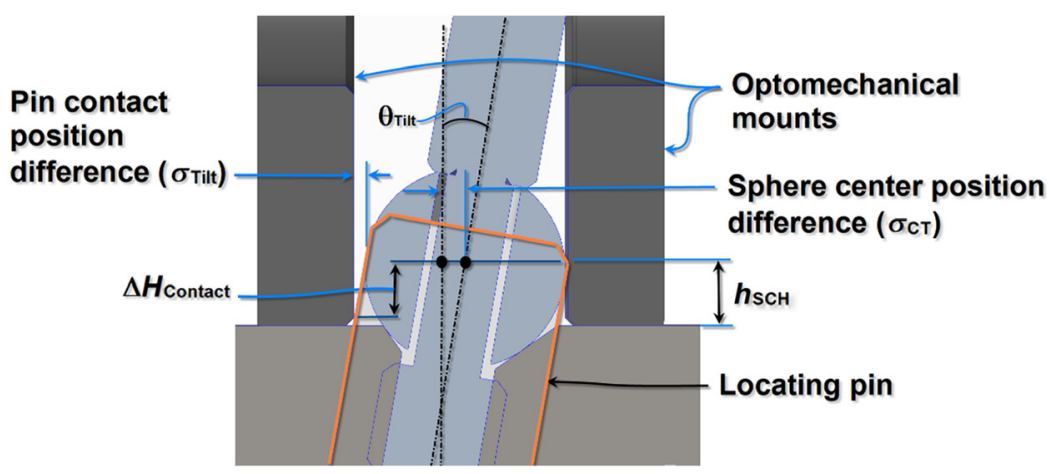

Fig. 4 Illustration of a hole tilting effect on position accuracy (angle magnified for visualization).

$$
\sigma_{\text {Tilt }}=\Delta H_{\text {Contact }} \cdot \tan \left(\theta_{\text {Tilt }}\right),
$$

where $\sigma_{\text {Tilt }}$ is the position error due to the pin tilt angle, $\Delta H_{\text {Contact }}$ is the contact height difference on the optomechanical mount when abutting from different directions, and $\theta_{\text {Tilt }}$ is the pin angle. $\Delta H_{\text {Contact }}$ is driven by the mount geometry but in the worst-case scenario it would be equal to the pin's height extruding of the breadboard. As mentioned, for the case on a tilted pin, the position uncertainty of a mount abutting on a tilted pin is the sum of $\sigma_{C T}$ and $\sigma_{T i l t}$. Figure 4 shows the optomechanical mount abutting position difference for a tilted pin compared to a ball when abutting from two different directions. Assuming $\theta_{\text {Tilt }}=0.25 \mathrm{deg}$ and $h_{\mathrm{SCH}} \cong \Delta H_{\text {Contact }}=$ $3 \mathrm{~mm}$ then from equations above we find $\sigma_{C T}=\sigma_{T i l t}=13 \mu \mathrm{m}$. Therefore, in this example, the position uncertainty of a mount abutting on the tilted pin would be $26 \mu \mathrm{m}$, essentially the double of the error expected for a sphere in a tilted cone with the same tilt error.

According to Machinery's Handbook, ${ }^{2}$ drilled holes accuracy is influenced by many factors such as accuracy of the drill point, the size of the drill, the work material, the length of the drill and the runout of the spindle and chuck assembly, and the rigidity of the machine tool to name a few. In this case, entry cone geometry can accommodate shorter and wider tools than threaded or pin holes of similar size, leading to stiffer tools and better position accuracy than tools required for deeper pin holes. Moreover, if the pins are removable, then some mechanical play must be accounted for between the pin and the hole to allow easy insertion/removal, which further increases the position uncertainty. The sum of these differences explains why spheres are more attractive for precise positioning than pins.

The position accuracy of an optical element does not depend only on the optomechanical mount position. The optical element position in its mount also needs to be controlled carefully to achieve accurate positioning in a multi-element system. The position error of a lens with respect to its optomechanical mount can greatly vary depending on the lens geometry. As discussed by Lamontagne et al., ${ }^{3}$ for self-centering to work the clamping angle criteria must be met, however, most long radius of curvature lenses [long focal lengths (FLs)] and negative (concave) lenses were still difficult to center as they often did not meet the auto-centering criteria or required custom-designed threaded rings and lens seats. With the integration of the patented edge contact mounting technique ${ }^{4}$ in the line of optomechanical mounts, it is possible to center most lenses without the need of custom-design threaded rings. The use of this technique, called QuickCTRedge mounting, allows a good lens position and tilt accuracy. For example, results from Lamontagne et al. ${ }^{1}$ in Table 1 display measured lens position accuracy with respect to cell rim using edge contact mounting.

To take advantage of these techniques, it was necessary to rethink the way the optomechanical mounts are constructed and used to maximize the position accuracy. Achieving high position accuracy "passively" can reduce the alignment process time by reducing the total number adjustable mounts required. Reducing the number of adjustable mounts can inevitably lower the total system weight on the breadboard and will also improve the behavior of the optical system when exposed to various vibrations levels.

It is understood that cases exist where optical components, opto-electronic components, or other need to be inserted in the optical system but do not have well defined (precise) reference 
Table 1 Centering measurements of a lens surface in contact with a threaded ring using edge contact mounting.

\begin{tabular}{lcccc}
\hline \hline \# of measurements & Min & Max & Mean & Std deviation \\
\hline 75 & $0.45 \mu \mathrm{m}$ & $18.62 \mu \mathrm{m}$ & $7.19 \mu \mathrm{m}$ & $4.72 \mu \mathrm{m}$ \\
94 & 0.04 arc min & 2.49 arc min & 0.81 arc min & 0.55 arc min \\
\hline \hline
\end{tabular}

features for accurate positioning, but yet require to be accurately positioned in the optical system (often at the beginning or the end of an optical branch of the system). For this purpose, a set of adjustable mounts compatible with the line of optomechanical mounts were designed. These mounts were designed with the same mechanical robustness philosophy and includes locking mechanisms suitable to survive similar environmental conditions. Once installed and aligned a first time, the adjustable mount provides the position accuracy features to the unprecise component to match with the line of optomechanical mounts.

Now, with the lens edge centering method combined with adequate mount fabrication tolerance controls and the addition of patent pending ball referencing features on the breadboards, ${ }^{5}$ it is expected to achieve improved optical element position accuracies under $100 \mu \mathrm{m}$ at the optomechanical mount level.

\subsection{Position Accuracy Aspects}

In the development of the line of optomechanical mounts, one important aspect of the design focused on what could be the achievable absolute and relative position accuracies. When assembled in a mount, the lens maximum position and tilt errors with respect to a theoretical optical path is the sum of all components (optical and optomechanical) fabrication errors and components interfacing errors. They are defined as position error contributors:

$$
\sigma_{\max }=\sum_{i=1}^{n} \sigma_{i}
$$

where $\sigma_{\max }$ is the sum of individual position error contributors $\sigma_{i}$ from the lens up to the system global coordinate system.

Table 2 displays a non-exhaustive list of possible errors sources (or contributors) to the lens position error and highlight typical error ranges expected for commercial grade components.

However, in practice, all forms of errors rarely occur in the same direction and at maximum value unless it is a systematic error. Therefore, a statistical approach often gives a better evaluation of the actual error stack-up. For this reason, the root-sum-squared method is often used:

$$
\sigma_{R S S}=\sqrt{\sum_{i=1}^{n} \sigma_{i}^{2}},
$$

where $\sigma_{R S S}$ is the square root of the sum of squared individual position error contributors $\sigma_{i}$ from the lens up to the system global coordinate system.

The $X, Y$ and $Z$ position accuracy of an optomechanical mount can be directly measured with respect to a given reference system. The total position accuracy in a plane or in volume is

$$
\begin{gathered}
A c c_{2 D}=\sqrt{A c c_{X}^{2}+A c c_{Y}^{2},} \\
A c c_{3 D}=\sqrt{A c c_{X}^{2}+A c c_{Y}^{2}+A c c_{Z}^{2},}
\end{gathered}
$$

where $A c c_{2 D}$ and $A c c_{3 D}$ are the scalar planar and volumetric position accuracies, respectively, and $A c c_{X}, A c c_{Y}$, and $A c c_{Z}$ are the components of position accuracy vector in mutually perpendicular axis. As shown in Fig. 5, the lens mounts angular errors are function of the 
Grenier et al.: Methods to achieve fast, accurate, and mechanically robust optical breadboard alignment

Table 2 Typical main contributors to optical element position error.

\begin{tabular}{|c|c|c|}
\hline Error form & Error range & Comment \\
\hline \multirow{3}{*}{$\begin{array}{l}\text { Manufacturing errors } \\
\text { of optical element }\end{array}$} & $\leq 50 \mu \mathrm{m}$ & Edge thickness difference of commercial-grade lenses ${ }^{6,7}$ \\
\hline & $\leq 100 \mu \mathrm{m}$ & Diameter of commercial-grade lenses ${ }^{6,7}$ \\
\hline & $\leq 150 \mu \mathrm{m}$ & Center thickness of commercial-grade lenses ${ }^{6,7}$ \\
\hline $\begin{array}{l}\text { Optical element residual } \\
\text { position error in its mount }\end{array}$ & $\leq 350 \mu \mathrm{m}$ & $\begin{array}{l}\text { Assuming a lens not self-centered, completely offset with } \\
\text { a lens to barrel play of } 350 \mu \mathrm{m}\end{array}$ \\
\hline $\begin{array}{l}\text { Manufacturing errors of the } \\
\text { lens/mirror seat position with } \\
\text { respect to the mount base or } \\
\text { abutment contact surface }\end{array}$ & $\leq 25 \mu \mathrm{m}$ & ISO 286 IT7 tolerance grade up to $50 \mathrm{~mm}$ feature size ${ }^{8}$ \\
\hline $\begin{array}{l}\text { Ball diameter error } \\
\text { (one ball versus the other) }\end{array}$ & $\leq 6 \mu \mathrm{m}$ & ISO 286 IT5 tolerance grade up to $10 \mathrm{~mm}$ feature size ${ }^{8}$ \\
\hline $\begin{array}{l}\text { Breadboard holes conical } \\
\text { interface position error with } \\
\text { respect to the others }\end{array}$ & $\leq 63 \mu \mathrm{m}$ & ISO 286 IT7 tolerance grade up to $500-\mathrm{mm}$ feature size ${ }^{8}$ \\
\hline $\begin{array}{l}\text { If applicable, manufacturing } \\
\text { errors of any additional parts } \\
\text { such as lens tube or mechanical } \\
\text { components located between the } \\
\text { lens and the mount }\end{array}$ & - & - \\
\hline
\end{tabular}

distance between the ball contact points in the horizontal plane and depends on the lens mount base width along the vertical and lateral plane. These angular errors also generate translations of the optical element that adds to the error stack up of to the reference sphere direct contact point position error and can be described as

$$
\begin{gathered}
\theta_{H}=\tan ^{-1}\left(\frac{\Delta z}{W_{B}}\right), \\
\theta_{V}=\tan ^{-1}\left(\frac{\Delta \varepsilon_{\perp}}{L_{B}}\right), \\
\theta_{L}=\tan ^{-1}\left(\frac{\Delta \varepsilon_{\perp}}{W_{B}}\right),
\end{gathered}
$$

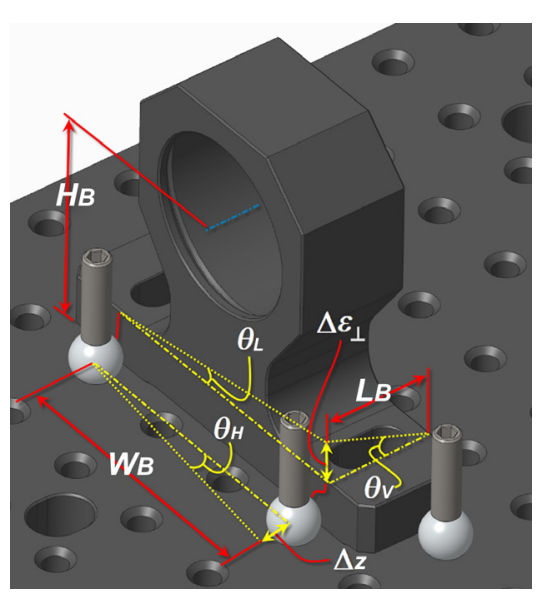

Fig. 5 Lens mount angular error definition. 


$$
\begin{gathered}
\delta_{A}=\frac{W_{B}}{2} \sin \theta_{H}+\frac{H_{B}}{2} \sin \theta_{V}, \\
\delta_{L}=H_{B} \sin \theta_{L}+\sigma_{L 3} \\
\delta_{V}=\frac{W_{B}}{2} \sin \theta_{L}
\end{gathered}
$$

where

- $\theta_{H}$ is the mount horizontal tilt angle (yaw angle).

- $\Delta z$ is the reference balls relative position difference in the normal direction to the abutting plane.

- $W_{B}$ is the distance between the reference the ball contact points in the abutting plane.

- $\theta_{V}$ is the mount vertical tilt angle (pitch angle).

- $\Delta \varepsilon_{\perp}$ is the height difference between the front and the back of the mount at mounting interface, this error generates a perpendicularity error of the mount.

- $L_{B}$ is the optomechanical mount thickness.

- $\theta_{L}$ is the mount horizontal tilt angle (roll angle).

- $H_{B}$ is the optomechanical mount nominal axis height.

- $\delta_{A}$ is the lens position error in the axis direction, resulting from abutting errors and angular deviations.

- $\delta_{L}$ is the lens translation in the perpendicular direction to the optical axis.

- $\sigma_{L 3}$ is the lateral abutting error of the optomechanical mount with the $3^{\text {rd }}$ sphere in the lateral axis.

- $\delta_{V}$ is the lens translation in the perpendicular direction to the breadboard plane, resulting from angular deviation.

Small rotations of the lens about its axis $\left(\theta_{L}\right)$ can be neglected as first-hand approximation for lenses with symmetry of revolution. These roll angle deviations of the mount due to flatness errors, or surface irregularities at the breadboard and mount interface would be measured as a lateral displacement of the optical element $(Y)$.

Therefore, it is possible to extract the different position and angular errors from simple threedimensional (3D) position sampling on an optomechanical mount. Then, for a given optomechanical mount with known lens seat position error and using Eqs. (8)-(13), it is possible to estimate the optical element position in space.

\section{Mechanical Robustness Considerations}

The position accuracy and repeatability can be valuable features for optomechanical mounts. However, to be able to use these optomechanical mounts and breadboards outside of the classical laboratory environment, it is safe to assume that vibrations levels are likely to be higher. The design parameters available to control and improve the vibration stability at the optomechanical mount level, are the mount stiffness $(k)$ and mass $(M)$, in other words, its stiffness to weight ratio. The optomechanical mount response to external vibration depends on the part's intrinsic natural resonance frequency $\left(f_{n}\right)$ as shown in Eq. (14).

$$
f_{n}=\frac{1}{2 \pi} \sqrt{\frac{\mathrm{k}}{M}} .
$$

The mount geometry is determinant in the control of this vibration response. This can be demonstrated by simplifying the optomechanical mount to a rectangular cantilever beam extruding from the breadboard surface and assuming it is rigidly fixed on it, the equation of $f_{n}$ becomes 


$$
f_{n} \cong \frac{1}{4 \pi} \sqrt{\frac{E \mathrm{~b} h^{3}}{M L^{3}}}
$$

where $E$ is the mount Youngs modulus, $b$ is the mount width perpendicular to the excitation axis and beam axis, $h$ is the mount thickness in the direction of the excitation axis (assuming the excitation axis is oriented in the mount's weakest orientation to obtain $b \geq h$ ), $M$ is the mount and lens assembly mass, and $L$ is the mount height (cantilever arm length). An optomechanical mount exposed to vibration excitation at or near its resonance frequency will amplify the amplitude of movement and acceleration to a point where holding forces to maintain an optical element could be exceeded. This can lead to optical system alignment loss. Therefore, designing the optomechanical mounts to have a high natural frequency is desirable to reduce or prevent misalignment to occur in transport.

Commercial transport random vibration spectrum can range from 0 to $2000 \mathrm{~Hz}$ according the minimum integrity exposure of department of defence test method standard (MIL-STD-810H). ${ }^{9}$ Therefore, the design of each element of the line of optomechanical mount included finite element analysis to optimize mount geometry to obtain high natural frequencies. The rationale behind the selection of this specific standard is, since these optomechanical mount have been designed to be used for several types of industrial applications, the minimum integrity exposure englobes most of worldwide shipping transportation vehicles.

\section{Experimental Validation}

The position accuracy and the position repeatability have two different implications when it comes to practical use. The position accuracy refers to the possibility to expect or predict where any optical element will be in space with respect to a theoretical (or perfect) layout. The repeatability refers to the ability to fall back in the same position for a given layout if an element needs to be removed and replaced.

Different experiments were performed to assess the achievable performance of the line of optomechanical mounts and breadboards:

1. Experiment 1: Position accuracy and repeatability measurement of multiple breadboards with reference spheres.

2. Experiment 2: Optomechanical mount position accuracy and repeatability measurement.

3. Experiment 3: Optical repeatability test.

4. Experiment 4: Environmental stability testing (temperature and vibration).

Material and environment:

For the experiments involving dimension probing, the experiments are performed in a clean room environment complying to ISO $\mathrm{N}$ class 7 standard $^{10}$ with a stable temperature of $21^{\circ} \mathrm{C} \pm 1{ }^{\circ} \mathrm{C}$. Optical and environmental testing is performed under normal laboratory conditions (ISO N class 8 to 9). Each experiment was performed over a short period of time extending from a few hours to less than a week. Environmental conditions in the laboratories during these experiments were stable. The components tested in the experiments are all from the line of breadboards and mounts named QuickPOZ, ${ }^{11}$ which include the position reference chamfers on each breadboard mounting hole and edge mount centering technology. Throughout the test campaign the components used were:

- Five different $200 \times 400 \times 12.7 \mathrm{~mm}^{3}$ breadboards,

- Six reference balls tools,

- Five fixed mirror mounts (25.4-mm diameter), height $31.8 \mathrm{~mm}$,

- One adjustable mirror mount (25.4-mm diameter), height $31.8 \mathrm{~mm}$,

- One lens mount (25.4-mm diameter), height $31.8 \mathrm{~mm}$,

- 1 threaded mount (25.4-mm diameter), height $31.8 \mathrm{~mm}$,

- 1 threaded lens tube (25.4-mm diameter), 12.7-mm length,

- Two $X Y$ adjustable mounts (25.4-mm diameter), height $25.4 \mathrm{~mm}$, 
- Two axial adjustment stages,

- Three edge centering rings for 25.4-mm diameter lenses.

Included optical components:

a. Four different 25.4-mm diameter lenses:

- About 50-mm FL doublet lens 47-637,

- 250-mm FL 63-845,

- 50-mm FL 49-356,

- 100-mm FL 47-641,

b. Five different $25.4 \mathrm{~mm}$ diameter flat mirrors BB1-E02.

Other optical hardware used included:

- 1 Sub miniature A fiber adapter,

- 1 UNF 1.035-40 to C-Mount thread adapter,

- 1 pigtailed monomode fiber laser source, 4- $\mu \mathrm{m}$ core, numerical aperture 0.12 ,

- 1 camera $1280 \times 1024$ pixels, $5.3-\mu \mathrm{m}$ pixel pitch.

\subsection{Reference Spheres Position Accuracy and Repeatability Experiment}

To assess the position accuracy of the breadboard's conical reference feature in combination with the reference spheres, experiment 1 is divided in two parts:

i. Experiment 1a: Position accuracy measurement of multiple breadboards with reference balls.

ii. Experiment $1 \mathrm{~b}$ : Reference ball assembly position repeatability tests.

The reference ball position accuracy and repeatability test over the breadboard surface consists in placing different reference balls in multiple positions on the breadboard, repeating the ball insertion and removal multiple times for each position, measuring the ball center position each time. The probing is executed with a Mitutoyo model BRT-710 Coordinate Measuring Machine (CMM) and the data acquisition and post-processing is done with Polyworks Metrology Suite software. ${ }^{12}$ The CMM is a precision position probing arm mounted on a three-axis motorized gantry. CMMs are metrology machines primarily used for quality control to verify dimensional conformance of fabricated goods. The end probe of the CMM arm is a calibrated sphere mounted on a force feedback extension rod. The CMM's probe makes small controlled contacts with the target component to measure the position coordinates at each contact point. By contacting multiple points on the reference sphere mounted in the breadboard's conical interface, it is possible to determine the reference sphere's center in 3D.

Method:

The breadboard is placed and secured in position on the CMM's granite table. The first sphere inserted on the breadboard is probed and its center serves as the coordinate system origin. Two other spheres are inserted on the breadboard and their centers serves as reference points to define the experiment reference axis directions. Using Polyworks software and a 3D computeraided design (CAD) model of the experiment as a nominal reference, a local coordinate system is defined with the probed reference points on the breadboard and a step by step probing sequence is defined. Figure 6 shows the general reference ball layout and axis definition for the experiment 1. At each position, the operator manually places the reference sphere on the predetermined position on breadboard and requests the CMM to probe the reference sphere's position for determining the relative position error. After the probing is completed, the sphere is removed, and the process is repeated for the next positions on the breadboard.

Experiment 1a: The tested breadboard dimensions are $200 \times 400 \times 12.7 \mathrm{~mm}^{3}$ with M4 threaded holes and positioning chamfers at a $12.5-\mathrm{mm}$ spacing. For each reference ball insertion, the sphere's center position is determined by the average calculation of 10 probed points around 


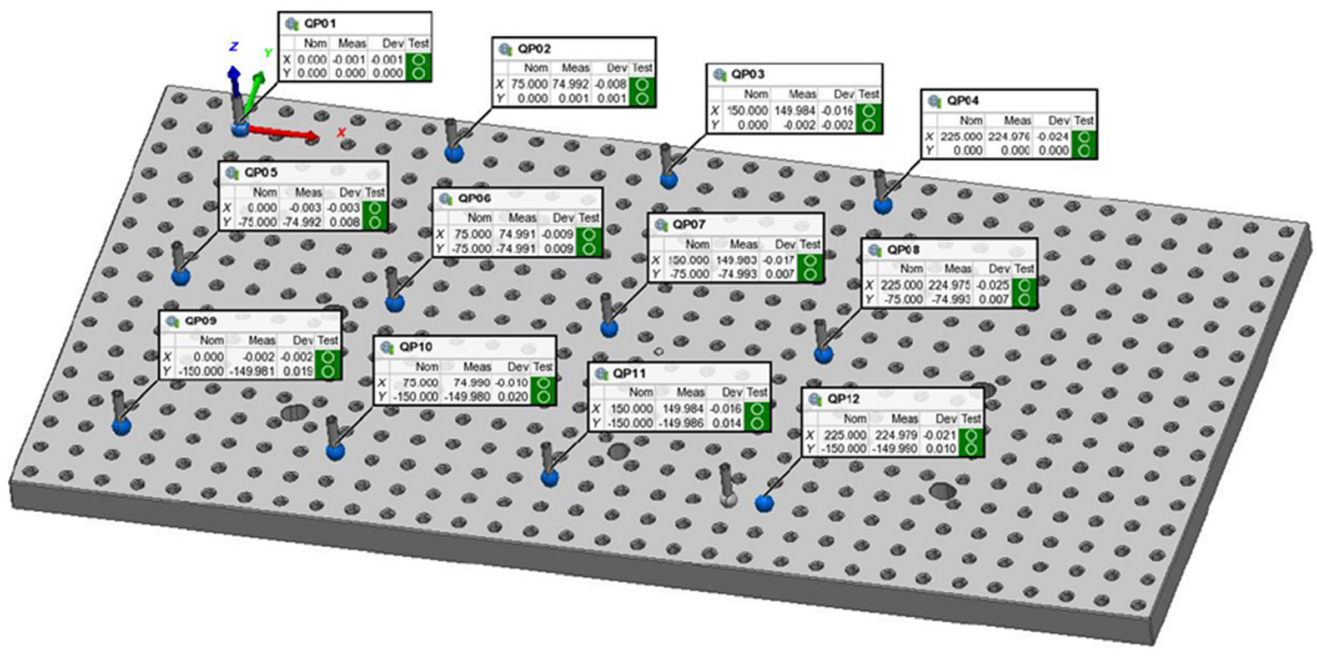

Fig. 6 Experiment 1 reference spheres position accuracy and repeatability experiment layout.

its external diameter. The position accuracy $\left(\operatorname{Acc}_{P}(\mathrm{x}, y, z)\right)$ and orientation accuracy $\left(\operatorname{Acc}_{O}\left(\theta_{V}, \theta_{H}, \theta_{\mathrm{L}}\right)\right)$ of the ball in a $3 \mathrm{D}$ space is evaluated as

$$
\begin{gathered}
A c c_{P}(x, y, z)=P_{T H}(\mathrm{x}, y, z)-P_{M}(x, \mathrm{y}, z), \\
A c c_{O}\left(\theta_{V}, \theta_{H}, \theta_{L}\right)=O_{T H}\left(\theta_{V}, \theta_{H}, \theta_{\mathrm{L}}\right)-O_{M}\left(\theta_{V}, \theta_{H}, \theta_{\mathrm{L}}\right),
\end{gathered}
$$

where $P_{T H}(x, y, z)$ is the theoretical position of the ball defined in the CAD model and $P_{M}(x, \mathrm{y}, z)$ is the measured position, and $O_{\mathrm{TH}}\left(\theta_{V}, \theta_{H}, \theta_{\mathrm{L}}\right)$ is the theoretical orientation of the ball defined in the CAD model and $O_{M}\left(\theta_{V}, \theta_{H}, \theta_{\mathrm{L}}\right)$ is the measured orientation. However, in this experiment the three rotations of the sphere are not useful due its symmetry of revolution and the height ( $Z$ axis) is not useful in the context of the breadboard positioning features. This leaves only the $X$ and $Y$ coordinates in the experiment $1 \operatorname{Acc}_{P}(x, y, z)$. The standard deviation is computed over the total accuracy measurements made along each axis.

Experiment $1 \mathrm{~b}$ : The reference ball assembly repeatability is evaluated by measuring an initial position and by comparing it with multiple assembly-removal operations on the same breadboard grid position. The process is then repeated at multiple locations on the breadboard. The position repeatability $\left(R P_{i}\left(x, y, z, \theta_{V}, \theta_{H}, \theta_{L}\right)\right)$ and angular repeatability $\left(A R_{i}\left(\theta_{V}, \theta_{H}, \theta_{L}\right)\right)$ are

$$
\begin{gathered}
R P_{i}(x, y, z)=P_{0}(x, y, z)-P_{j}(x, y, z), \\
A R_{i}\left(\theta_{V}, \theta_{H}, \theta_{L}\right)=O_{0}\left(\theta_{V}, \theta_{H}, \theta_{L}\right)-O_{j}\left(\theta_{V}, \theta_{H}, \theta_{L}\right),
\end{gathered}
$$

where $P_{0}(x, y, z)$ and $O_{0}\left(\theta_{V}, \theta_{H}, \theta_{L}\right)$ are the initial measured position and angles respectively for a given tested position $(i)$, and $P_{j}(x, y, z)$ and $O_{j}\left(\theta_{V}, \theta_{H}, \theta_{L}\right)$ are the repeated position and rotations measurement ( 1 to $j$ ) for its given position. Once again, in this case only the $X$ and $Y$ values are useful for the experiment thus only those values will be monitored.

\subsection{Optomechanical Mount Position Accuracy and Repeatability Experiment}

Experiment 2: To assess the position accuracy of the optomechanical mount, the experiment is also divided in two parts:

i. Experiment 2a: Optomechanical mount position accuracy measurement

ii. Experiment 2b: Optomechanical mount positioning repeatability measurement

The optomechanical mount accuracy and repeatability tests performed with the same principle than in experiment 1, equations (16 to 19), but measurements are made in three axes $(X, Y, Z)$ instead of two and two rotations $\left(\theta_{V}, \theta_{H}\right)$. The optomechanical mount is inserted and 


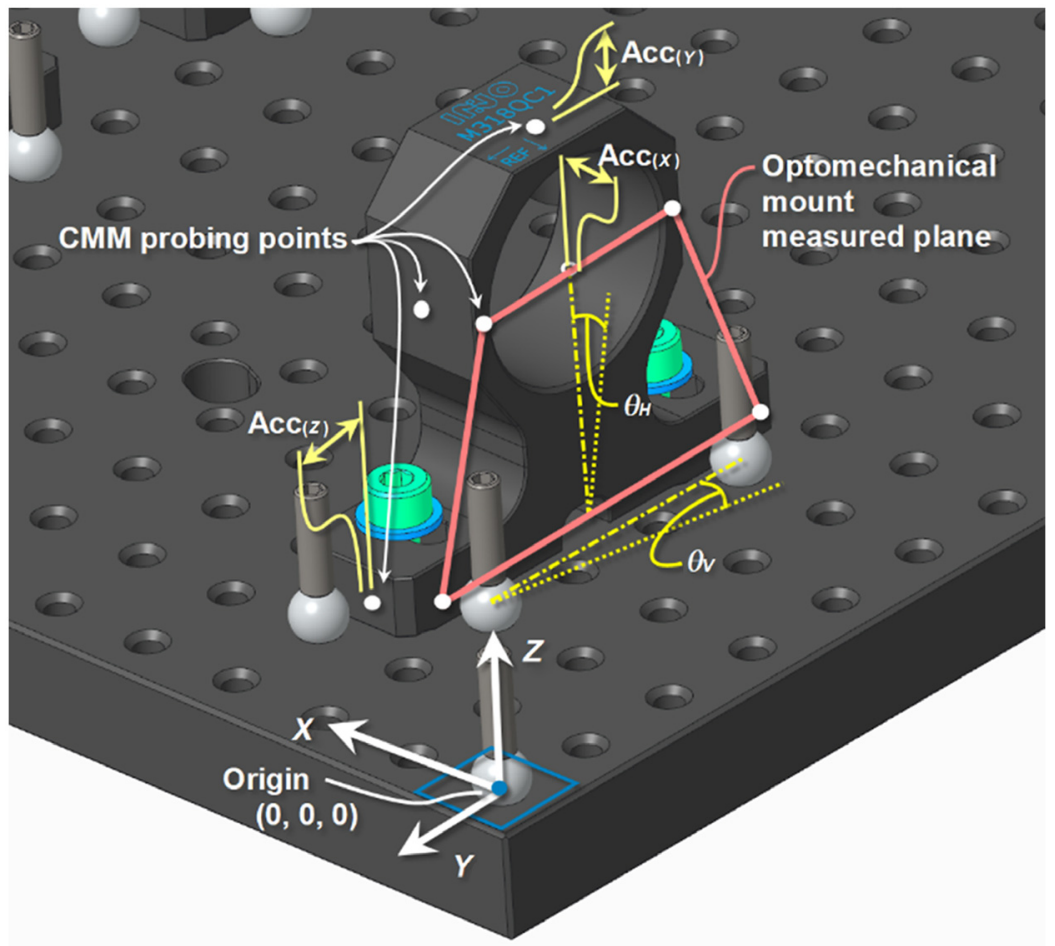

Fig. 7 Experiment 2 optomechanical mount test layout and probing scheme.

removed multiple times in each position after the three reference balls are positioned properly for a specific lens mount position. For each mount insertion, the mount is installed on the breadboard and lightly pressed by hand against the three reference spheres and fixed in place with two M4 screws. The $X, Y$, and $Z$ positions are measured with the CMM, by probing the mount at seven distinct location for each three sides (see Figure 7). The associated tip and tilt errors $\left(\theta_{V}, \theta_{H}\right)$ are extracted from the face probing of the mount. The deviation in $Z$ and $X$ axis for each mount is retrieved from the top and side probing of the optomechanical mount respectively. The $X$ axis deviation is computed at the lens center's height from the plane formed by the four probing points on the mount face plan. For each optomechanical mount measurement, the probing is done through a programmed and automated pattern to ensure measurement consistency.

\subsection{Optical Repeatability Experiment}

A similar validation can be done optically by inserting and removing optical elements of a simple optical assembly one at the time. In this experiment, labeled Experiment 3, a fiber laser source is collimated through a 50-mm FL lens and sent through two folding mirrors to a 250-mm FL lens and then imaged on a 5.3- $\mu \mathrm{m}$ pixel pitch camera, as shown in Figure 8. The imaging lens and camera combination can detect small lens and mirror movements (decenters and tilts) by

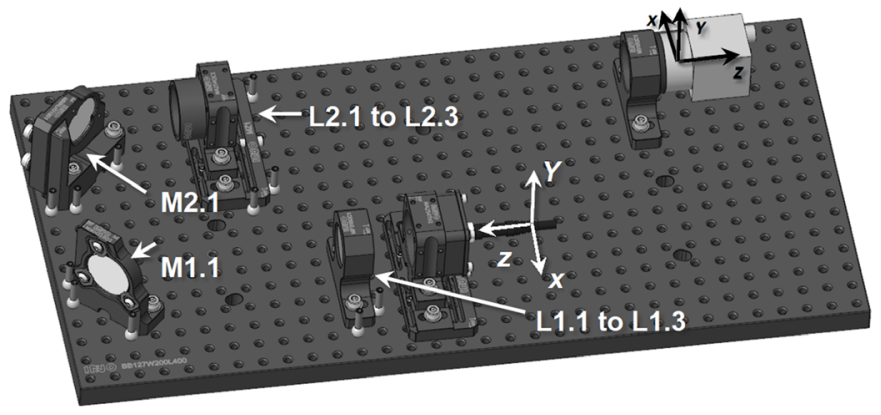

Fig. 8 Experiment 3 optical repeatability test. 
measuring the beam centroid position in pixels. For a given initial beam position on the camera, each optomechanical mount is removed and reassembled using the reference balls. The beam centroid position is logged each time.

The lens optical repeatability test is divided by type of optomechanical mounts (lens mount, mirror mount, and lens tubes) and by interfaces (reference spheres, and edge centering) to measure the individual and combined effect of these interfaces on the position repeatability:

Lens mount 1 (L1):

- L1.1: Removing the optomechanical mount only and placing it back in position with the help of reference balls.

- L1.2: Removing lens mount, loosening the lens retaining ring, tightening it back in position, and placing the mount back in position with the help of reference balls.

Mirror mount 1 (M1):

- M1.1: Removing the optomechanical mount M1 only and placing it back in position with the help of reference balls.

Mirror mount 2 (M2):

- M2.1: Removing the optomechanical mount M2 only and placing it back in position with the help of reference balls.

Lens mount 2 (L2):

- L2.1: Removing the optomechanical mount L2 only and placing it back in position with the help of reference balls.

- L2.2: The lens tube is removed and reassembled back in position

- L2.3: The lens tube and lens retaining ring are removed and reassembled back in position

In addition to the lens self-centering functions already discussed in Sec. 4, the lens tubes are also designed to be self-centered when inserted in other mounts of the family, or in a tube to tube configuration. Tests on lens mount 2 are aimed to confirming the combined repeatability of lens and tube's self-centering. These cases represent typical conditions in the laboratory where it is required to remove a mount or a component to access a hard to reach component nearby or to require inspection or cleaning.

The centroid computation is done at $1 / 10$ 'th of pixel in resolution and the system jitter was measured at $\sim \pm 1 / 2$ pixel. The beam centroid movement noted at the camera can be configured to different translation or angle scaling factors depending if it is a lens or a mirror and according to the focal lens ratio. Therefore, for each optical element, its specific movement can be related to beam centroid translation on the sensor:

$$
\begin{gathered}
\delta_{\text {Sensor }}=\operatorname{Pix}_{P} \sqrt{\Delta C E N_{X}^{2}+\Delta C E N_{Y}^{2}}, \\
\delta_{\text {SenX }}=\operatorname{Pix}_{P} \cdot \Delta C E N_{X}, \\
\delta_{\text {SenY }}=\operatorname{Pix}_{P} \cdot \Delta C E N_{Y}, \\
\Delta L_{1}=\delta_{\text {Sensor }} \cdot\left(\frac{F L_{1}}{F L_{2}}\right), \\
\Delta L_{2}=\delta_{\text {Sensor }}, \\
\theta_{X}=\frac{1}{2} \tan ^{-1}\left(\frac{-\delta_{\text {SenX }}}{F L_{2}}\right),
\end{gathered}
$$




$$
\theta_{Y}=\frac{1}{2} \tan ^{-1}\left(\frac{\delta_{\operatorname{Sen} Y}}{\cos \frac{\pi}{4} \cdot F L_{2}}\right)
$$

where

- $\delta_{\text {Sensor }}$ is the centroid deviation measured at the sensor,

- Pix $_{P}$ is the pixel pitch $(5.3 \mu \mathrm{m})$.

- $\triangle C E N_{X}$ is the pixel count of the centroid deviation on the sensor in $X$.

- $\triangle C E N_{Y}$ is the pixel count of the centroid deviation on the sensor in $Y$.

- $\delta_{\text {SenX }}$ is $\delta_{\text {Sensor }}$ component of the measured displacement in the $X$ direction.

- $\delta_{\text {SenY } Y}$ is $\delta_{\text {Sensor }}$ component of the measured displacement in the $Y$ direction.

- $\Delta L_{1}$ is the first lens deviation in $X$ or $Y$ (optic fiber side).

- $L_{1}$ - is the first lens conversion factor (optic fiber side).

- $F L_{1}$ is the first lens focal length $(50 \mathrm{~mm})$.

- $F L_{2}$ is the second lens focal length $(250 \mathrm{~mm})$.

- $\Delta L_{2}$ is the second lens deviation in $X$ or $Y$ (camera side).

- $\theta_{X}$ is the angular deviation of a mirror about the $X$ axis.

- $\theta_{Y}$ is the angular deviation of a mirror about the $Y$ axis.

Lateral translations of the mirrors about the $X$ and $Y$ axis (parallel to the mirror plane) do not affect the beam position on the sensor unless the displacement is wide enough to create beam clipping, which should not occur for small displacements. $Z$ axis translations of the mirrors (normal to the mirror plane) create a translation of the optical axis but do not affect beam position on the sensor for small displacements and can also be neglected if no beam clipping occurs. Thus, for mirror mounts, only angular deviations will be noticeable in this experiment. Small angular deviations of the lens mounts, or of the lens in relation to its mount, will produce effects similar to the lens translation in the $X$ and $Y$ axis (normal to the optical axis) and will be undistinguishable from the lens mount translations at the detector level. Lens mount translations about the $Z$ axis (parallel to the optical axis) create defocus at the detector level, which would affect the spot size on the detector and should not affect the centroid position unless the spot shape on the detector is also altered. This is not expected to occur for small translations. For this reason, only translations are considered for the lens mounts in the experiment, but the measured translation can either be the result of a translation or an angular movement of the lens or a combination of both. The measured lens position deviation can be converted to an equivalent lens surface tilt $(\theta)$ if needed, using Eq. (27):

$$
\theta=\sin ^{-1}\left(\frac{\Delta L_{i}}{R o C_{i}}\right)
$$

where the $\Delta L_{i}$ is $\Delta L_{1}$ or $\Delta L_{2}$ and $R o C_{i}$ is the optical surface radius in contact with the lens mount seat of lens 1 or 2 . In this case, the radius of doublet lens 1 (surface 1) is $34.53 \mathrm{~mm}$ and the radius of lens 2 is $228.73 \mathrm{~mm}$.

The Eqs. (20)-(26) can be simplified to extract a fixed conversion factor for each optical element as shown in Table 3.

It can be noted that mirror horizontal and vertical angular motions have different scaling factors. This is related to the mirror mounts being placed at $45 \mathrm{deg}$ with respect to the incoming beam in one axis and at $90 \mathrm{deg}$ in the other direction. The mechanical angle deviation of the mount is doubled at the camera along the sensor horizontal axis but is multiplied by $\sqrt{2}$ in the sensor vertical axis. It should also be noted that an angular motion of M1 or M2 mirror along their axis parallel to the breadboard horizontal plane produces a coupled deviation at the camera in $X$ and $Y$. An optical modeled representing the experiment bench (Fig. 9) was generated using OpticStudio software ${ }^{13}$ and utilized to calculate this effect. As shown in Fig. 10, an angular deviation of $\sim 0.2 \mathrm{deg}$ about M2 $X$ axis will cause a beam motion at the sensor of $\sim 1.2 \mathrm{~mm}$ in $Y$ (vertical) but only $\sim 3 \mu \mathrm{m}$ in $X$ (horizontal). Hence, the coupling effect is small and is neglected in the analysis. 


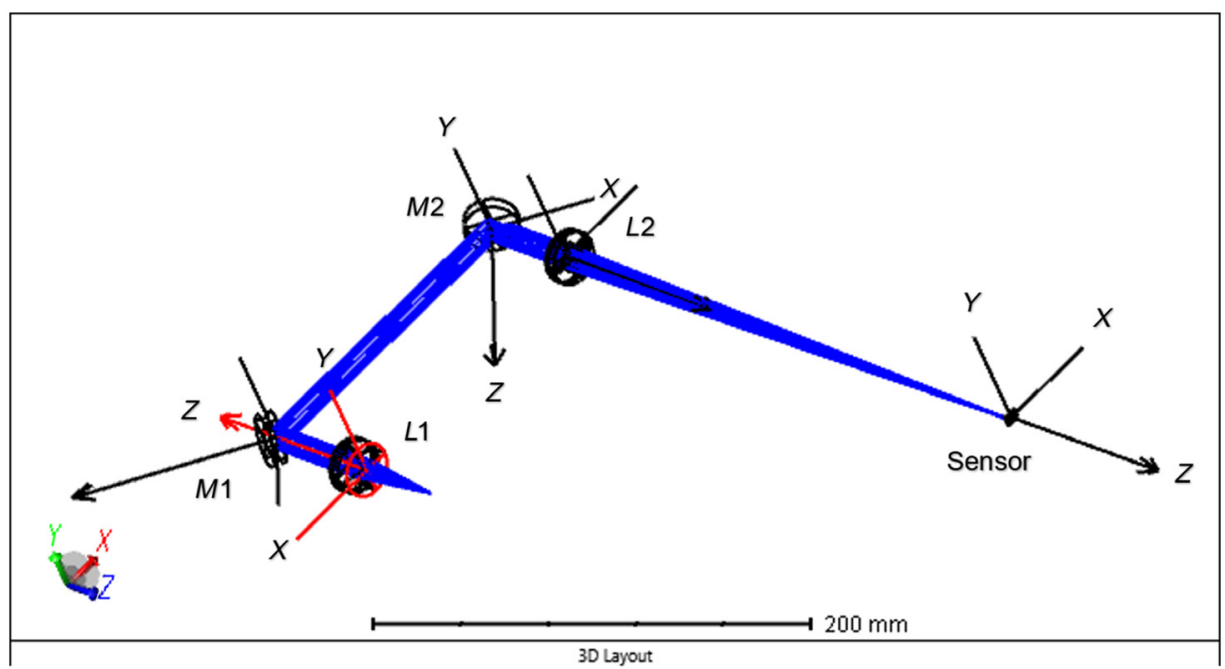

Fig. 9 Experiment 3 optical layout with optical element and axes definition.

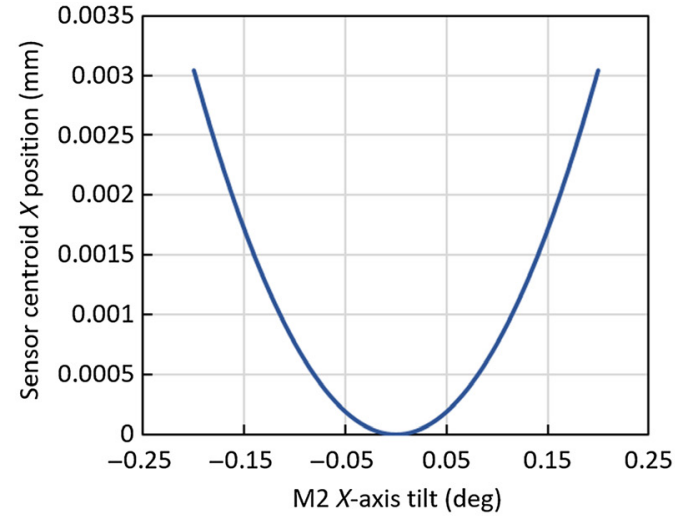

(a)

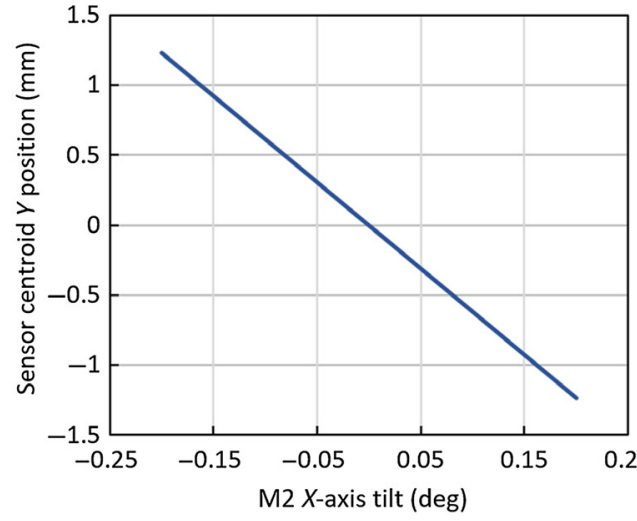

(b)

Fig. 10 Sensor plane centroid position in (a) $X$ and (b) $Y$ versus M2 tilting angle in local $X$ axis orientation. Refer to Fig. 9 for local axes definition.

\section{Experiment 4: Environmental Stability}

To extend the usefulness of breadboard optical assemblies, in addition to the position accuracy and repeatability improvements, the mechanical stability under changing environmental conditions is also an important aspect that was considered in the optomechanical mount design. It should be worth that such high accuracy mounts may withstand shipping standards. A vibration and thermal test campaign were performed with the breadboard optical assembly to verify its capacity to survive severe environmental conditions.

In these experiments, an optical assembly comparable to that of the optical repeatability test is used. However, in this case, the collimated beam between the two lenses is folded five times to increase the sensitivity of the assembly to misalignment, as shown in Fig. 11. The lens combination used for the test is different to allow more space for the folding mirrors: the second lens is a shorter FL of $100 \mathrm{~mm}$ lens instead of the $250 \mathrm{~mm}$ FL of the experiment 3. The conversion factors based on Eqs 18-21 of the experiment are listed in Table 4:

The breadboard assembly is cycled seven times from $+25^{\circ} \mathrm{C}$ to $+65^{\circ} \mathrm{C}$ and one time at $-46^{\circ} \mathrm{C}$ for $24 \mathrm{~h}$ as shown in Fig. 12, without any shipping packaging, in accordance with MIL-STD$810 \mathrm{H}$ standard, using method 501.7 and 502.7 - Procedure I. ${ }^{9}$

The optical bench assembly was used without the shipping box for the thermal cycling. This is considered a worst-case scenario since packaging acts as a thermal insulator, which increases 


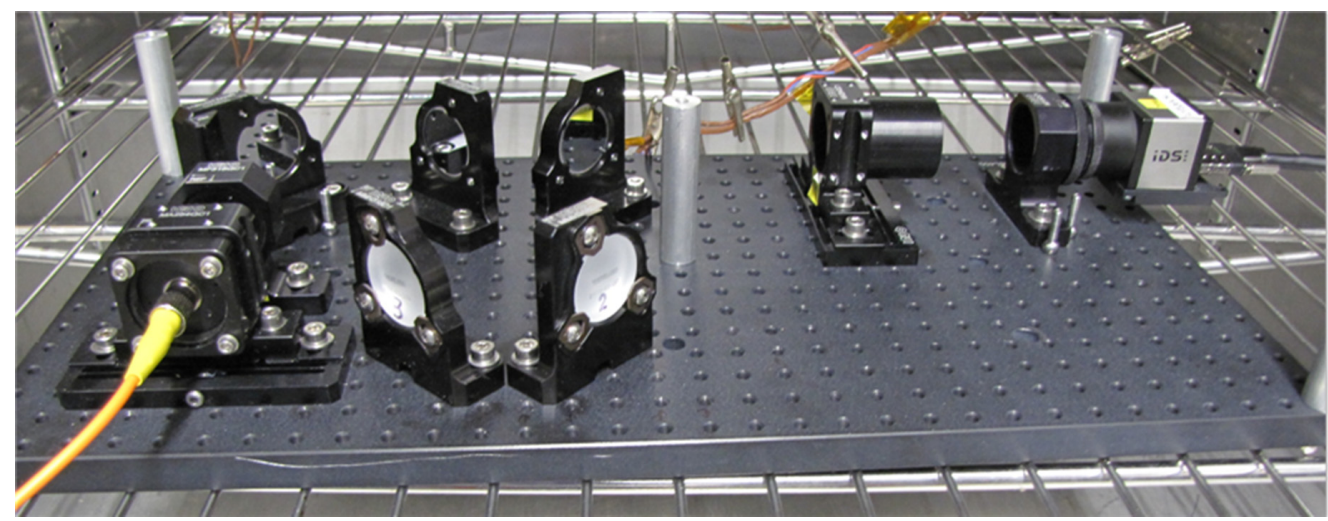

Fig. 11 Experiment 4 environmental stability test setup.

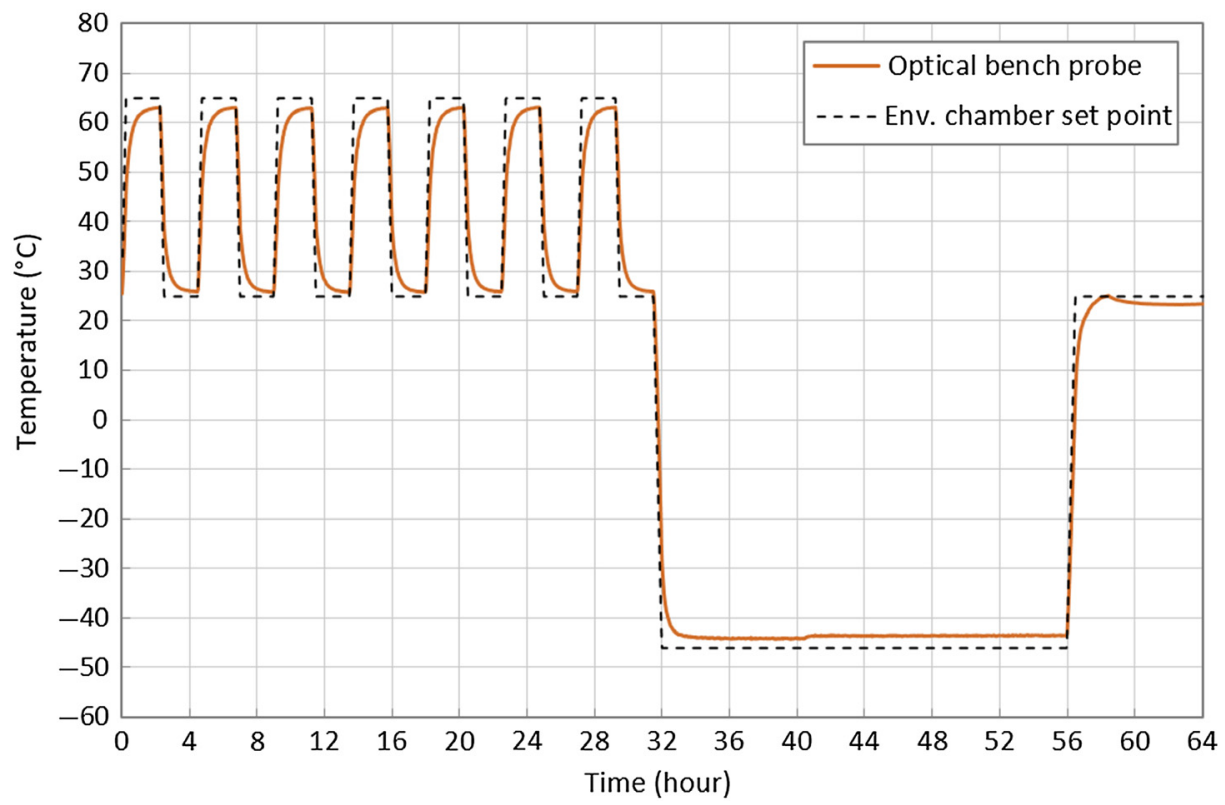

Fig. 12 Experiment 4 thermal cycles profile.

the time needed to reach thermal equilibrium and can possibly reducing the total excursion range viewed by the optical system in short duration exposition to extreme temperatures.

For the vibration campaign a general random vibration minimum integrity exposure (Fig. 13) as per MIL-STD- $810 \mathrm{H}$ method $514.8^{9}$ of $7.7 \mathrm{Grms}, 20$ to $2000 \mathrm{~Hz}, 1 \mathrm{~h}$ per axis, was selected.

For the purpose of the vibration test, a 1.6-mm-thick sheet metal plate was mounted on the five $12.5-\mathrm{mm}$ lab posts shown in Fig. 11 to serve as an emulated top cover. The assembly was then wrapped with two layers of 12.7-mm-thick polyethylene air bubble sheets. The wrapped assembly was then placed centered in an evenly distributed 50-mm-thick layer of styrofoam nuggets inside cardboard box. The fibered laser source was disconnected from the lab setup for the packaging, and it was noted that it introduces a centroid jitter of about \pm 3 pixels in the measurements.

\section{Experimental Results}

The results of the four experiments are summarized in Tables 5-7. It is important to note that the coordinate systems defined in the CMM probing experiments (experiment 1 and 2) are different than the ones defined in optical repeatability test experiments (experiment 3 and 4). Axes definition for the CMM experiments ( 1 and 2) do not share the same origin but do share similar axes 


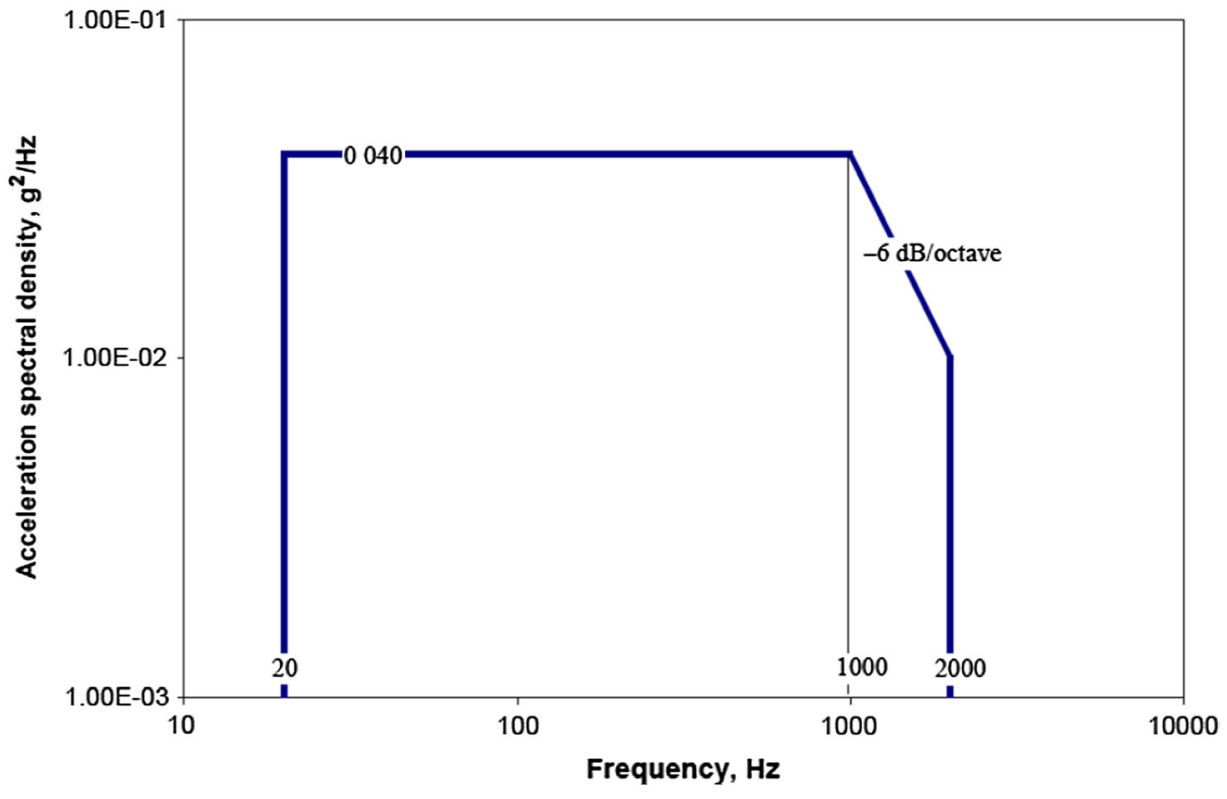

Fig. 13 Experiment 4 random acceleration profiled used in the vibration test. ${ }^{9}$

Table 3 Experiment 3 conversion factors.

\begin{tabular}{lcc}
\hline \hline & \multicolumn{2}{c}{ Conversion factor } \\
\cline { 2 - 3 } Optical element & $X$ (horizontal) & $Y$ (vertical) \\
\hline$L_{1}$ & $1.06 \mu \mathrm{m} /$ pixel & $1.06 \mu \mathrm{m} / \mathrm{pixel}$ \\
$L_{2}$ & $5.3 \mu \mathrm{m} /$ pixel & $5.3 \mu \mathrm{m} / \mathrm{pixel}$ \\
$M_{1}$ & $10.6 \mu \mathrm{rad} /$ pixel & $14.99 \mu \mathrm{rad} / \mathrm{pixel}$ \\
$M_{2}$ & $10.6 \mu \mathrm{rad} / \mathrm{pixel}$ & $14.99 \mu \mathrm{rad} / \mathrm{pixel}$ \\
\hline \hline
\end{tabular}

Table 4 Experiment 4 conversion factors.

\begin{tabular}{lcc}
\hline \hline \multirow{2}{*}{ Description } & \multicolumn{2}{c}{ Conversion factor } \\
\cline { 2 - 3 } & $X$ (horizontal) & $Y$ (vertical) \\
\hline$L_{1}$ & $2.5 \mu \mathrm{m} /$ pixel & $2.5 \mu \mathrm{m} /$ pixel \\
$L_{2}$ & $5.3 \mu \mathrm{m} /$ pixel & $5.3 \mu \mathrm{m} / \mathrm{pixel}$ \\
$M_{1}$ to $M_{5}$ & $26.5 \mu \mathrm{rad} /$ pixel & $37.5 \mu \mathrm{rad} / \mathrm{pixel}$ \\
\hline \hline
\end{tabular}

orientations. However, experiment 3 and 4 share the same coordinate system philosophy: $Z$ axis follows the optical path. Therefore, results from CMM probing and optical validations cannot be compared directly axis to axis.

\subsection{Results Experiment 1 and 2}

For the CMM probing experiments the, the coordinate system is fixed and aligned with the breadboard hole grid as defined in Figs. 6 and 7. The results of the Table 5-Experiment 1 and 2 CMM probing results displays the accuracy and repeatability measured in individual axis. 
Table 5 Experiment 1 and 2 CMM probing results.

\begin{tabular}{|c|c|c|c|c|c|c|c|}
\hline Test & & Direction & MIN & MAX & $\begin{array}{c}2 \sigma \\
\text { Deviation }\end{array}$ & $\begin{array}{l}\text { Measurement } \\
\text { Uncertainty }\end{array}$ & $\begin{array}{c}\text { \# of } \\
\text { samples }\end{array}$ \\
\hline \multirow{4}{*}{$\begin{array}{l}\text { Experiment } 1 \\
\text { reference } \\
\text { sphere and } \\
\text { breadboard }^{\mathrm{a}}\end{array}$} & \multirow[t]{2}{*}{$\begin{array}{l}\text { Position Accuracy } \\
\qquad\left(\operatorname{Acc}_{P}(x, \mathrm{y})\right)\end{array}$} & $x$ & $-25 \mu \mathrm{m}$ & $39 \mu \mathrm{m}$ & $33 \mu \mathrm{m}$ & $\pm 5 \mu \mathrm{m}$ & 72 \\
\hline & & Y & $-9 \mu \mathrm{m}$ & $20 \mu \mathrm{m}$ & $13 \mu \mathrm{m}$ & $\pm 5 \mu \mathrm{m}$ & 72 \\
\hline & \multirow{2}{*}{$\begin{array}{l}\text { Position Repeatability } \\
\qquad\left(R P_{i}(x, y)\right)\end{array}$} & $x$ & - & - & $3 \mu \mathrm{m}$ & $\pm 5 \mu \mathrm{m}$ & 24 \\
\hline & & $Y$ & - & - & $2 \mu \mathrm{m}$ & $\pm 5 \mu \mathrm{m}$ & 24 \\
\hline \multirow{10}{*}{$\begin{array}{l}\text { Experiment } 2 \\
\text { Optomechanical } \\
\text { mount }\end{array}$} & \multirow{3}{*}{$\begin{array}{l}\text { Position Accuracy } \\
\qquad\left(A c c_{P}(\mathbf{X}, \mathbf{z})\right)\end{array}$} & $x$ & $-31 \mu \mathrm{m}$ & $-2 \mu \mathrm{m}$ & $20 \mu \mathrm{m}$ & $\pm 5 \mu \mathrm{m}$ & 22 \\
\hline & & $Y$ & $0 \mu \mathrm{m}$ & $33 \mu \mathrm{m}$ & $28 \mu \mathrm{m}$ & $\pm 5 \mu \mathrm{m}$ & 22 \\
\hline & & $Z$ & $0 \mu \mathrm{m}$ & $58 \mu \mathrm{m}$ & $47 \mu \mathrm{m}$ & $\pm 5 \mu \mathrm{m}$ & 22 \\
\hline & \multirow{3}{*}{$\begin{array}{l}\text { Position Repeatability } \\
\quad\left(R P_{i}(x, y, z)\right)\end{array}$} & $x$ & - & - & $2 \mu \mathrm{m}$ & $\pm 5 \mu \mathrm{m}$ & 22 \\
\hline & & Y & - & - & $2 \mu \mathrm{m}$ & $\pm 5 \mu \mathrm{m}$ & 22 \\
\hline & & $z$ & - & - & $1 \mu \mathrm{m}$ & $\pm 5 \mu \mathrm{m}$ & 22 \\
\hline & \multirow[t]{2}{*}{$\begin{array}{l}\text { Angular Accuracy } \\
\left(\operatorname{Acc}_{O}\left(\theta_{V}, \theta_{H}, \theta_{\mathrm{L}}\right)\right)\end{array}$} & $\begin{array}{c}X Z \\
\text { plane }\left(\theta_{V}\right)\end{array}$ & $-541 \mu \mathrm{rad}$ & $-297 \mu \mathrm{rad}$ & $159 \mu \mathrm{rad}$ & - & 22 \\
\hline & & $\begin{array}{c}X Y \\
\text { plane }\left(\theta_{H}\right)\end{array}$ & $-35 \mu \mathrm{rad}$ & $122 \mu \mathrm{rad}$ & $82 \mu \mathrm{rad}$ & - & 22 \\
\hline & \multirow[t]{2}{*}{$\begin{array}{l}\text { Angular Repeatability } \\
\qquad\left(A R_{i}\left(\theta_{V}, \theta_{H}\right)\right)\end{array}$} & $\begin{array}{c}X Z \\
\text { plane }\left(\theta_{V}\right)\end{array}$ & - & - & $47 \mu \mathrm{rad}$ & - & 22 \\
\hline & & $\begin{array}{c}X Y \\
\text { plane }\left(\theta_{H}\right)\end{array}$ & - & - & $48 \mu \mathrm{rad}$ & - & 22 \\
\hline
\end{tabular}

aBased on reference ball position measurements over a surface of $15 \times 225 \mathrm{~mm}^{2}$ on four different breadboards $200 \times 400 \mathrm{~mm}$.

Table 6 Experiment 3 optical repeatability results.

\begin{tabular}{|c|c|c|c|c|c|c|c|c|}
\hline \multirow[b]{2}{*}{ Description } & \multicolumn{2}{|c|}{ Minimum } & \multicolumn{2}{|c|}{ Maximum } & \multicolumn{2}{|c|}{$2 \sigma$ deviation } & \multirow{2}{*}{$\begin{array}{c}\text { Measurement } \\
\text { uncertainties }\end{array}$} & \multirow{2}{*}{$\begin{array}{c}\text { \# of } \\
\text { samples }\end{array}$} \\
\hline & $x$ & $Y$ & $x$ & $Y$ & $x$ & $Y$ & & \\
\hline $\begin{array}{l}\text { L1 mount only } \\
\text { (L1.1) }\end{array}$ & $-0.4 \mu \mathrm{m}$ & $0 \mu \mathrm{m}$ & $0.3 \mu \mathrm{m}$ & $0.7 \mu \mathrm{m}$ & $0.6 \mu \mathrm{m}$ & $0.7 \mu \mathrm{m}$ & $\pm 0.5 \mu \mathrm{m}$ & 5 \\
\hline $\begin{array}{l}\text { L1 mount and lens } \\
\text { ring loosen (L1.2) }\end{array}$ & $-2.5 \mu \mathrm{m}$ & $-0.2 \mu \mathrm{m}$ & $3.7 \mu \mathrm{m}$ & $1.4 \mu \mathrm{m}$ & $4.8 \mu \mathrm{m}$ & $1.3 \mu \mathrm{m}$ & $\pm 0.5 \mu \mathrm{m}$ & 5 \\
\hline M1 mount (M1.1) & $0 \mu \mathrm{rad}$ & $0 \mu \mathrm{rad}$ & $6.7 \mu \mathrm{rad}$ & $5 \mu \mathrm{rad}$ & $5.5 \mu \mathrm{rad}$ & $4 \mu \mathrm{rad}$ & $\begin{array}{l}\mathrm{Y}: \pm 5.3 \mu \mathrm{rad} \\
\mathrm{X}: \pm 7.5 \mu \mathrm{rad}\end{array}$ & 5 \\
\hline M2 mount (M2.1) & $-33.1 \mu \mathrm{rad}$ & $-15.5 \mu \mathrm{rad}$ & $0 \mu \mathrm{rad}$ & $0 \mu \mathrm{rad}$ & $25.7 \mu \mathrm{rad}$ & $11.9 \mu \mathrm{rad}$ & $\begin{array}{l}\mathrm{Y}: \pm 5.3 \mu \mathrm{rad} \\
\mathrm{X}: \pm 7.5 \mu \mathrm{rad}\end{array}$ & 5 \\
\hline $\begin{array}{l}\text { L2 mount only } \\
\text { (L2.1) }\end{array}$ & $0.1 \mu \mathrm{m}$ & $-6.5 \mu \mathrm{m}$ & $8.9 \mu \mathrm{m}$ & $3.9 \mu \mathrm{m}$ & $7.2 \mu \mathrm{m}$ & $7.9 \mu \mathrm{m}$ & $\pm 2.6 \mu \mathrm{m}$ & 5 \\
\hline $\begin{array}{l}\text { L2 lens tube only } \\
\text { (L2.2) }\end{array}$ & $-2.8 \mu \mathrm{m}$ & $-1 \mu \mathrm{m}$ & $-0.2 \mu \mathrm{m}$ & $4.6 \mu \mathrm{m}$ & $2.1 \mu \mathrm{m}$ & $5.3 \mu \mathrm{m}$ & $\pm 2.6 \mu \mathrm{m}$ & 5 \\
\hline $\begin{array}{l}\text { L2 lens tube and } \\
\text { lens loosen (L2.3) }\end{array}$ & $0.2 \mu \mathrm{m}$ & $-4.3 \mu \mathrm{m}$ & $3.8 \mu \mathrm{m}$ & $6.4 \mu \mathrm{m}$ & $3 \mu \mathrm{m}$ & $8.1 \mu \mathrm{m}$ & $\pm 2.6 \mu \mathrm{m}$ & 5 \\
\hline
\end{tabular}


Table 7 Environmental stability results in experiment 4.

\begin{tabular}{lccccccc}
\hline \hline & \multicolumn{2}{c}{ Deviation $(\mathrm{px})$} & & \multicolumn{2}{c}{ Deviation $(\mu \mathrm{m})$} & & Uncertainty \\
\cline { 2 - 3 } Test description & $X$ & $Y$ & & $X$ & $Y$ & & $\begin{array}{c}\text { Amplitude } \\
(\mu \mathrm{m})\end{array}$ \\
\hline & 1 & -1 & & 5.3 & -5.3 & \pm 2.6 & 7.5 \\
Thermal cycling & 1 & 3 & & 15.9 & 15.9 & \pm 15.9 & 22.5 \\
\hline Random vibration & -3 & &
\end{tabular}

From the results of experiment 1, it is possible to see that the position accuracy $\left(\mathrm{Acc}_{2 D}\right)$ for the reference sphere's position is about $35 \mu \mathrm{m}$ at $2 \sigma$. From experiment 2 , the results show that it is possible to achieve precise positioning of an optomechanical mount over the tested surface with values under $66 \mu \mathrm{m}$ and $180 \mu \mathrm{rad}$ at $2 \sigma\left(\mathrm{Acc}_{3 D}\right)$ with respect to its theoretical position. The position repeatability measured is very low throughout the experiment at an average $\pm 2 \mu \mathrm{m}$ at $2 \sigma$, which is below the CMM absolute uncertainty. The CMM error envelope is within $\pm 5 \mu \mathrm{m}$ at $2 \sigma$ over the measurement envelope. Therefore, the optomechanical mount repeatability cannot be ruled better than the CMM absolute error in these experiments. Although, even if it seems probable that the repeatability is better than the CMM's uncertainty of $5 \mu \mathrm{m}$, it will be assumed to be $\pm 5 \mu \mathrm{m}$. It is assumed that the use of automated CMM probing is significant in the high repeatability monitored, by consistently probing the same regions of the optomechanical mounts therefore reducing the effect of surface irregularities. The angular accuracy measured in the vertical plane is $<160 \mu \mathrm{rad}(33 \mathrm{arcsec})$ at $2 \sigma$. This is slightly higher than the one measured in the horizontal plane of $82 \mu \mathrm{rad}$. This was expected since the distance between the points of contact of the reference balls on optomechanical mount in the horizontal axis is longer than the depth of the optomechanical mount in the perpendicular axis. A small deviation at the mount interface with the breadboard as a greater effect in the vertical axis than a similar deviation in the horizontal axis.

\subsection{Results Experiment 3 Optical Repeatability}

The experiment 3 results are displayed in Table 6 . The axis definition of this experience the axis to be at the optical element as shown in Fig. 9 and the values should be interpreted as the optical element displacement or rotation.

The magnitude of displacement measured in the optical repeatability test in the $X Y$ plane (perpendicular to the optical axis) is $8.6 \mu \mathrm{m}$ at $2 \sigma$ for experiment (L2.1) and $10.7 \mu \mathrm{m}$ for experiment (L2.3). Considering the number of contact interfaces involved in each of this experiment sub steps, the results seems close to the $\pm 5 \mu \mathrm{m}$ repeatability per interface noted in experiment 2 . It can also be noted that the two mirror mounts have slightly different behaviors, M1 at magnitude of $6.8 \mu \mathrm{rad}$ appears to be more than two times more stable than M2 at $28.3 \mu \mathrm{rad}$. This is in accordance with the theory since M2 is an adjustable mount that is heavier and has more interfaces than M1, which is a fixed mount. However, it should be noted that throughout the experiment the deviations monitored were close to the centroid total jitter/uncertainty of the optical setup that was noted in the lab and no signal averaging was done. For this reason, the repeatability should not be considered better than $\pm 1 / 2$ pixel, which would mean at least $\pm 0.5 \mu \mathrm{m}$ for the 50 -mm lens, $\pm 2.6 \mu \mathrm{m}$ for the 250 -mm lens, $\pm 5.3 \mu \mathrm{rad}$ ( $Y$ axis tilt), and $\pm 7.5 \mu \mathrm{rad}$ ( $X$ axis tilt) for the mirrors. The sampling is five trials per subtest step for a total of 35 measurements

Comparing to the standard lab post visual alignment case discussed in introduction, the results of experiment 1,2 , and 3 suggest that the combination of the edge centering technique with the breadboard's conical reference features and spheres technique in this line of breadboards and optomechanical mounts have the potential to improve position accuracy of an optical element by a factor of 10. It could still be required to use adjustable mounts if the alignment is required to be lower than $50 \mu \mathrm{m} / 179 \mu \mathrm{rad}$ and to compensate for components that do not possess reference features, but in most cases, the number of adjustable mounts can be reduced thus possibly reducing total alignment time. 


\subsection{Results Experiment 4}

After the vibration campaign, the beam centroid was compared to the initial value and a total displacement amplitude of 4.2 pixels or $22.5 \mu \mathrm{m}$ of the centroid was observed as shown in Table 7 and no physical damage was noted. Based on conversions factors of Table 4, it could be assumed to be either a single mirror mount movement of $79.5 \mu \mathrm{rad}$ in horizontal and $112.5 \mu \mathrm{rad}$ in vertical or, more likely, a distributed movement over multiple mirrors for an average of $16 \mu \mathrm{rad} /$ mirror and $22.5 \mu \mathrm{rad} /$ mirror in each axis. Considering the small lateral play present around the 25.4-mm diameter mirrors used and the mirror clamping method, a small sliding of the mirror with respect to the optomechanical mount interface can produce a similar angular deviation due to surface roughness. In example, assuming the mirror mounting pads are $17.5 \mathrm{~mm}$ apart, a small surface irregularity of $0.35 \mu \mathrm{m}$ is sufficient to produce a $20 \mu \mathrm{rad}$ mirror deviation. In this case, the movement is assumed to be related to the mirrors since the clamping method of mirrors may allow some sliding to occur at the mirror/mount interface whereas a lens/ ring combination is less likely to produce large displacements. If the same movement is to be related to a lens decenter, then it would either be a displacement of $22 \mu \mathrm{m}$ of Lens \#2 or $10.5 \mu \mathrm{m}$ for lens \#1. However, this is assumed unlikely since the optical repeatability test results showed better repeatability values than $10 \mu \mathrm{m}$ in all cases. This would imply that a ring would have loosen, yet visual inspection of the test specimens made after the test did not show any loose component. Once again, it should be noted that the measured values are of the same amplitude than the uncertainty of the test setup. For many applications this would be considered small movements confirming that the technology is suitable to survive shipping conditions.

\section{Conclusions}

This paper presented a method to passively align optomechanical assemblies by uniting edge contact mounting techniques with a new patent pending optomechanical mount referencing technique, consisting of inserting reference spheres in a laboratory breadboard configuration. The operation principle of the sphere referencing method mount was presented and some of the advantages of this positioning technique in regard of the position accuracy were presented with respect to the locating pin method along with a brief overview of the self-centering capabilities of the edge contact mounting technique. Optomechanical mounts position and angular accuracy aspects were discussed and some of the typical error sources were quantified. The line of optomechanical mounts design driving factors to improve mechanical robustness were discussed in regard of the environmental conditions expected to occur in shipping. The position accuracy, repeatability, and mechanical robustness were demonstrated by two direct CMM probing experiments and two indirect optical experiments. The position repeatability measured for the reference sphere's positioning method, the edge contact mounting and with the optomechanical mount abutment on reference spheres were measured to be about $\pm 5 \mu \mathrm{m}$ per interface. The results show that it is possible to achieve position accuracies under than $50 \mu \mathrm{m}$ at $2 \sigma$ in two dimensions, or under $66 \mu \mathrm{m}$ and $180 \mu \mathrm{rad}$ at $2 \sigma$ in $3 \mathrm{D}\left(\mathrm{Acc}_{3 D}\right)$, at the optomechanical mount level for small transportable optomechanical assemblies. In comparison, to the laboratory post visual alignment case, the results suggest that the presented line of optomechanical mount and breadboards can improve by a factor of 10 the initial positioning of optical elements. The mechanical robustness of a typical breadboard level optical system was also demonstrated for temperature range from $+65^{\circ} \mathrm{C}$ down to $-46^{\circ} \mathrm{C}$ and at vibration ranges typical of that what should be encountered for worldwide shipping. The line of optomechanical mounts and breadboards developed with these methods, named QuickPOZ, opens the possibility to expand usage of laboratory setups to small productions due to reduced alignment time and built-in robustness.

\section{Acknowledgments}

The authors would like to thank INO for the financial support and to address special thanks to Mathieu Tremblay, Jacques Régnier, Marco St-Pierre, Mélanie Leclerc, and Martin Otis for their support in the test campaign. 


\section{References}

1. Carr Lane Manufacturing Co., "Catalog LXIII, Section 6: locators," https://publications .virtualpaper.com/steadyrain/clm_catalog_lxiii_locators/\#2/(accessed November 2020).

2. E. V. Oberg, Machinery's Handbook, 25th ed., p. 855, Industrial Press Inc., New York (1996).

3. F. Lamontagne et al., "Lens centering using edge contact mounting," Proc. SPIE 11103, 1110304 (2019).

4. M. Savard and F. Lamontagne, "Centering of an optical element using edge contact mounting," U.S. Pat. Appl. US20200264402A1 (2020).

5. M. Grenier et al., "Methods and optomechanical kits for building optical systems using self-aligned reference balls," U.S. Pat. Appl. US202063086797P (2020).

6. R. E. Fischer et al., Optical System Design, McGraw-Hill, New York (2000).

7. Optimax Systems Inc., "Optimax manufacturing tolerance chart," https://www.optimaxsi .com/optical-manufacturing-tolerance-chart (accessed January 2021).

8. International Organization for Standardization, "Geometrical product specifications (GPS) - ISO code system for tolerances on linear sizes-Part 1: Basis of tolerances, deviations and fits," ISO 286-1:2010, 2010, https://www.iso.org/obp/ui/\#iso:std:iso:286:-1:ed-2: v1:en.

9. United States Department of Defense, "Department of Defense Test Method Standard: Environmental Engineering Considerations and Laboratory Tests," MIL-STD-810H, Washington DC (2019).

10. International Organization for Standardization, "Cleanrooms and associated controlled environments-Part 1: classification of air cleanliness by particle concentration," ISO 14644-1:2015, 2012, https://www.iso.org/obp/ui/\#iso:std:iso:14644:-1:ed-2:v1:en.

11. INO, "QuickPOZ-Optomechanical mounts and breadboards 2020-2021 catalog," Document \#INO-PLT0201-0037 version 3.0, 2020, https://inostorage.blob.core.windows .net/media/2267/quickpoz-catalog.pdf (accessed December 2020).

12. Polyworks Metrology Suite, Version 2019 IR9, InnovMetric Software Inc., Quebec, QC (2019).

13. OpticStudio, Version 20.3, Zemax LLC, Kirkland, WA (2021).

Martin Grenier is a senior optomechanical engineer with over 20 years of experience, holding a bachelor's degree in mechanical engineering from Laval University. He has participated in the development of complex optical systems for military, security, industrial, astronomy, medical, and aerospace applications in his career. He worked extensively in the design and qualification of laser systems (IR to DUV), telescopes, and zooms used in harsh environmental conditions.

Nichola Desnoyers holds a master's degree in optomechanical engineering and a bachelor's degree in mechanical engineering from Laval University. He participated in the development of more than sixty optical systems for astronomy, military, security, medical, aerospace, industrial, telecommunication, and environmental applications at INO. He is a senior optomechanical engineer with over 25 years of experience more specifically in dimensional stability, thermal management, athermal structures, and optomechanical CFRP structures.

Frederic Lamontagne is a senior optomechanical engineer with over 19 years of professional experience. He has been involved in the development of complex optical systems for astronomy, space, military, medical, scientific, and industrial applications. He has authored a chapter and coauthored two chapters on optomechanical tolerance analysis and lens mounting in the second edition of the Handbook of Optomechanical Engineering. He has also authored 15 technical papers and has six awarded and five pending patents.

Bruno Leduc holds a bachelor's and a master's degree in mechanical engineering from Laval University. He cumulated 10 years of professional experience in mechanical design, R\&D, and FEA consulting. In the past five years, his work was dedicated to optomechanical design at INO, where he contributed to the development of optical systems for astronomy, military, medical, industrial, robotics, and airborne applications. His fields of expertise are optomechanical STOP analyses, dimensional stability, thermal management, and CFRP structures and adhesives. 
Mathieu Legros is an optomechanical engineer holding a bachelor's degree in mechanical engineering from Laval University. He developed expertise related to precise optical assemblies, thermal management, tolerance analysis, and athermal structures with over 13 years of professional experience related to optomechanics. He performed the design and the numerical simulations (FEA) of several types of complex optical systems for aerospace, military, astronomy, medical, security, and industrial applications.

Simon Paradis received his bachelor's and master's degrees in mechanical engineering from Laval University. His main expertise is in the field of numerical simulation (FEA \& CFD analysis) and product development. He is currently working in the field of optomechanical engineering with a focus on disruptive technologies. 